\title{
Clothes4all: A Novel Resource for Studying and Improving Web Accessibility
}

\author{
Till HALBACH ${ }^{\mathrm{a}, 1}$ and Vegard HAUGSTVEDT ${ }^{\mathrm{b}}$ \\ ${ }^{\mathrm{a}}$ Norwegian Computing Center (nr.no) \\ ${ }^{\mathrm{b}}$ Webstep AS (webstep.no)
}

\begin{abstract}
We present a novel and versatile online resource named Clothes4all which is a website, web application, and tool likewise, and which can be used to study various aspects of web accessibility. The article elaborates on the tool's development, its features and possibilities, as well as use and potential methodology. Clothes4all mimics a web shop for clothes and consists as such of a set of coherent web pages that can be freely used, studied, and extended as desired, in particular aiming at user trials. The site's main feature is that single or multiple accessibility barriers can be injected into or removed from its web pages in a controlled manner. The primary application area of Clothes4all is the testing and validation of accessibility checkers and validators themselves, and hereby the site is expected to eventually contribute to more accessible web pages. A secondary application area is education, as Clothes4all is a great resource to learn about web technologies, web accessibility, assistive technology, user diversity, impairments, and other aspects of online accessibility.
\end{abstract}

Keywords. Web/online A11Y, WCAG, accessibility failures, website, tool

\section{Introduction}

Web/online accessibility, sometimes abbreviated as A11Y, is about making web resources technically accessible to all people, regardless of peoples' age, gender, cultural background, IT literacy, impairments (including temporal and situational impairments), technical devices, and similar [1]. Progress regarding web accessibility is, among others, driven by a special group of the World Wide Web Consortium, the W3C's Web Accessibility Initiative [2]. One of the tasks of the W3C WAI is the development and publication of international recommendations / guidelines for web content accessibility, abbreviated as WCAG. The first guidelines, WCAG 1, were published in 1999 [3], and in 2008 there was a major update with WCAG 2.0 [4]. At the time of writing, there is WCAG 2.1 with 78 so-called success criteria in total, divided into 13 guidelines, four principles, and three different levels [5].

Basically, web accessibility is about giving everybody an equal opportunity to participate in the digital life [6]. Using Internet banking, shopping, consuming media, communicating with each other, and interacting with public services online is something most people take for granted today. It is therefore crucial that everyone has access to and can use these services, particularly in the light of the fact that between 
$15 \%$ and $19 \%$ of the world population are people with disabilities / impairments [7]. "People with impairments" is a quite heterogeneous group, though, which often is divided according to the three major categories sensor, motor, and cognition [8]. More fine-grained categories exist, e.g. [9]. The statistics are mirrored in national and international legislation: Non-discrimination, full and effective participation and inclusion in society, respect for difference and acceptance of persons with disabilities as part of human diversity and humanity, equality of opportunity, and accessibility (among others) are all guiding principles of the Convention on the Rights of Persons with Disabilities (CRPD), which most countries have ratified as of today [10].

To reach the goal of highly accessible web resources, web developers, testers, designers, researchers, and the like rely heavily on digital means to support them. However, they also need the proper education and training to learn about web accessibility, suitable tools, and how to use them. The web site presented here targets all these areas. It forms a basis for learning about the diversity of users, web accessibility basics, how to test for accessibility, and about the impact of various web accessibility measures and code and design choices.

The main contribution of this article is to present this novel web resource, to elaborate on its technical details, and how it can be used in web development, testing, and education. The remaining article is structured as follows: After a brief discussion of related solutions, we explain what methods we have employed to develop this web resource, and its technical details and design choices are described in detail. Then, the results from a limited trial with users with and without various impairments are presented, before the article concludes, together with an outlook of planned work and potential future ways.

\section{Related solutions}

To our knowledge, there are only a couple of related solutions which are similar to this novel tool.

The demo site offered by the $\mathrm{W} 3 \mathrm{C}$ perhaps is closest to what we have developed here [11]. It consists of a small number of contextually interrelated pages which are available both with and without accessibility flaws. One of its advantages is that there is an overview of injected errors and possible solutions. Its design, however, has not been updated for quite a while, and it lacks a modern appearance and modern technologies such as Javascript, dynamic content, responsive design, and similar. The site's content has become old-fashioned, too, and it is easy to spot that this is no real news site, rendering the site as difficult to employ in user trials as described further below. The site does not support multiple languages, either, and it is not possible to turn on and off single accessibility errors.

The British Governmental Digital Services have collected a number of accessibility failures (143 to be exact) for testing purposes as part of gov.uk, all combined in one single page [12]. Their focus is on the (deliberately injected) failures, but not on technical good solutions, and the page lacks examples of such, the possibility for before/after comparisons, the context of multiple pages and an entire site, and can therefore not be used in user trials. Also, the page is available in English only. 
The Norwegian IT company MediaLT has developed a series of pages with examples for good solutions [13]. In contrast to the aforementioned solutions, they have focused on single technical components and single failures, so their solution lacks a site's context, and the possibility for before/after comparisons. Besides, it is only available in Norwegian, which excludes international users.

There is another group at the W3C, the ACT Rules Community, which have done related work [14]. They have authored a number of accessibility tests with corresponding test cases and good and bad examples, which - among others - can be used to validate tools. The scope of their work, however, is the establishment of a set of rules, and as such there is no complete website which could be employed in user trials. All test cases contain only minimal markup snippets and exist only in English.

\section{Method, technical choices $\&$ description of resource}

We have developed a website that can be used to study various aspects of web accessibility, as detailed in the following. Due to its wide range of application, it is also denoted here as web application, web service, or simply tool. The list of requirements is based on an analysis of the deficiencies of the related solutions, and the input from user groups as detailed further below.

The development was embedded in a user-centered process, with university staff (lecturers, students) as the primary target group, as the site is expected to be used in courses at respectively University of Oslo and Oslo Metropolitan University. The staff were presented prototypes of increasing maturity in multiple iterations, and their feedback was incorporated in the development. The site's secondary user group are ordinary users, both with and without impairments. We therefore also had a trial with 11 users of the categories low vision, reduced motor (cerebral palsy and others), dyslexia, non-native speakers, combinations of the aforementioned, and no impairments. The users were asked to solve small tasks such as "switch to Norwegian", "what is the cost of delivery to your place", and "buy two products of your choice with the appropriate size, including customer registration and payment".

In order to be able to conduct such tasks, the site consists of a number of clothing products of various categories, such as coats, pants, shirts, and so on, for both males and females, and with a product count high enough for the site to be taken as an ordinary online store for clothes. The site was dubbed Clothes4all and is available under the domain name clothes4all.net. At the time of writing, there are 12 product pages, including provisions for five different product sizes, and several general pages with content like "Terms", "Delivery", and "About", and others. As the site tries to mimic an ordinary online store, it offers a shopping cart and a rudimentary checkout of orders, where personal details and payment details have to be filled in. The site supports multiple languages, and so far English and Norwegian are available.

Clothes4all is built as a React application running on Gatsby/NodeJS. One of our goals was to prove that it is possible to create accessible websites using modern technology, and this has driven the technology choices, going for modern, popular technologies, without making the learning curve for contributors too steep. Other than these frameworks, we did not want to have too many dependencies from third-party components, so we have relied on building parts and components from the bottom up as much as possible. As such, the code base itself can serve as an example of how to 
develop accessible components, and these components can nicely be extracted into a component library that can be browsed, downloaded, and used by anyone, as detailed in the outlook.

As a way to avoid privacy issues related to data input by the user, we only store data using session storage in the browser, meaning no user data is transmitted to the server. Because the website also works as a Single-Page Application (SPA) and is built on Gatsby, i.e. a static site generator, the site does not need a backend at all. This in turn makes the site quick to load and eliminates the need to require a lot of resources for the site to run. Many hosting services provide free hosting for static sites, like Github Pages and Netlify, and Clothes4all is currently hosted on the latter.

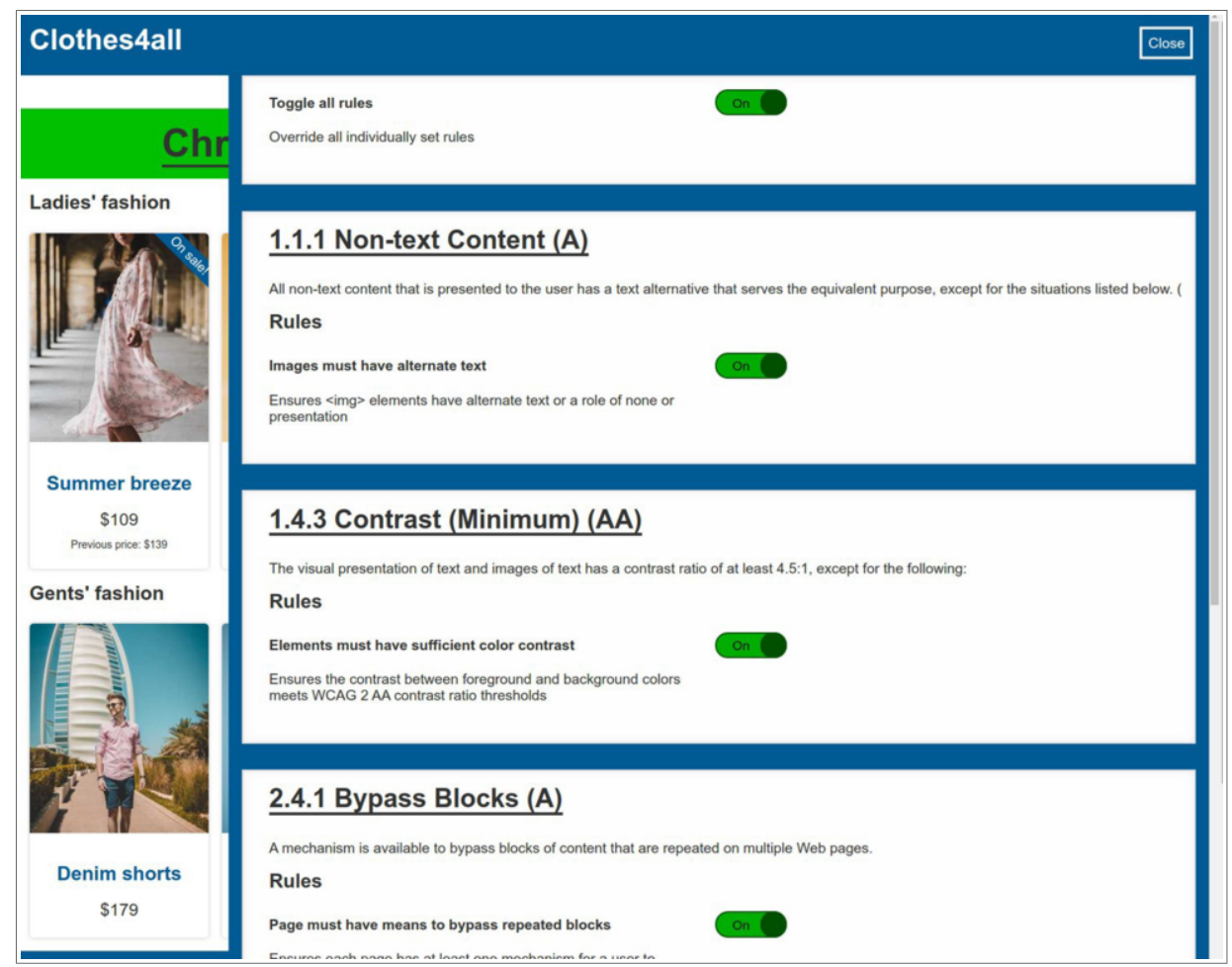

Figure 1. Screenshot of Clothes4all's slide-in side bar with rule controls

All content is stored in configuration files, and the application chooses the correct language file based on the browser's country code, falling back to English if no suitable language string is provided. Having version control and storage of all translations in the code base eases outside contributions through pull requests and lowers the threshold for translating content into new languages, as compared to architectures where content is stored in a Content Management System (CMS) with separate access and version control.

The main feature of Clothes4all is that it can be configured to contain or be free of accessibility errors in a controlled manner. Accessibility flaws in turn correspond to selected WCAG criteria, and flaw injection is here dubbed rule. There is a slide-in component in the graphical user interface (UI), see Figure 1, where single rules can be 
turned on and off; "on" means that the site complies with a particular WCAG criterion, ergo no corresponding accessibility flaws, and "off" means that accessibility flaws breaking that particular criterion are injected in the site, in all components and pages. For instance, WCAG Criterion 3.3.2 at Level A requires labels for user input, and with the corresponding rule enabled, <label $>$ elements are correctly used and coded in HTML with, say, input elements such as <input type="text">, whereas they are missing if this rule is disabled. At the time of writing, the site has implemented five such rules covering 1.1.1 Non-text content, 1.4.3 Minimum contrast, 2.4.1 Bypass blocks, 3.1.1 Language of page, and 3.3.2 Labels or instructions. It is stressed that a page must contain relevant elements for a rule to have an effect. As an example, the rule for page language affects all pages, but the rule for labels only affects pages with input field elements. For convenience, all rules can be turned on/off by a single UI switch, to switch between completely accessible and completely inaccessible versions of the site. This switch is also available programmatically with a HTTP parameter, such that it is possible to link to either the accessible or inaccessible version.

To wrap up, the tool's benefits are as follows:

- It can be used to give meaningful tasks in user trials.

- It has a modern appearance.

- It integrates modern web technologies such as HTML5, Javascript, and responsive design.

- It is built up by means of localized and descriptive content files in the code base to ease translations, modifications, and the addition of new content.

- It allows direct comparisons of a page's accessible version with the inaccessible version.

- It allows to turn on/off single accessibility flaws.

- It is multilingual.

- It is freely available as open source under the MIT licence and can thus be used and extended by anyone.

We also want to stress the advantages of Clothes4all for educators: The tool can be used to learn about web technologies in general and about the technical sides of web accessibility like markup, style, document object model, etc. in particular. It is also ideal to learn about related areas, such as technical recommendations like WCAG and ARIA, tools like checkers and validators, and assistive technology like screen readers and magnification. One of the site's main strengths, though, is the demonstration of the impact of accessibility measures on websites. Some measures have a visual impact, others not. For example, turning off the "1.4.3 Minimum contrast" rule will give an insufficient contrast ratio for selected page elements as shown in Figure 2. On the contrary, turning off "1.1.1 Non-text content" will suppress the alt attributes for image elements, which does not change the rendering in the graphical browser, but the effect will be noticeable in a screen reader. As such, the site can be used to study in detail what technological circumstances comprise a barrier for particular assistive technologies, and potentially how the user experience changes. The site is hence well suited to learn about and create empathy for people with impairments.

Besides strictly educational topics, Clothes4all targets web development and testing contexts in form of the injection of accessibility flaws into the site's web pages in a controlled manner to see whether or not they are detected by accessibility checkers and in accessibility audits. This is especially useful to determine potential false positives and false negatives, as well as to study whether the accessibility failures are properly detected as true positives. Another area of application is that Clothes4all may 


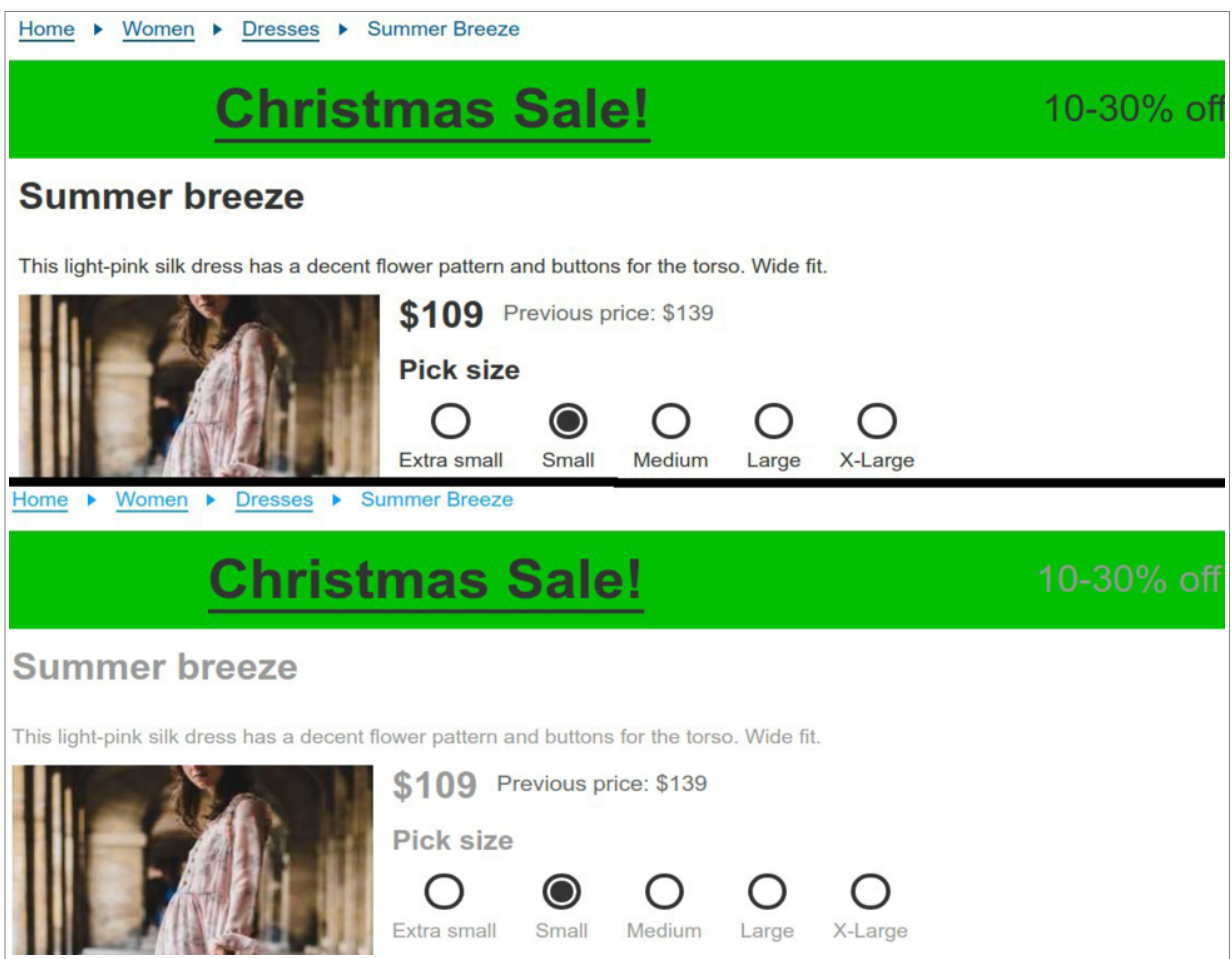

Figure 2. Screenshots of Clothes4all with contrast rule enabled (top) and disabled (bottom)

serve as a baseline in user trials and accessibility / usability evaluations. A given audit metric, say SUS (see further below), can be calculated for Clothes4all with and without certain flaws, as well as for various versions of the particular solution that needs to be assessed, by letting users from various user groups test both as part of task assignments. The metric's different values can then be compared with each other. This enables comparisons of technical solutions and multiple versions of them, as well as comparisons of user groups. It also enables the calculation of severity of particular accessibility issues, say missing alt attributes for images. It is further possible to compute multiple metrics and compare their fitness for the purpose of audits.

\section{User trials, results, and limitations}

As mentioned before, we conducted a user trial with individuals from multiple user groups to gain feedback on the site, and to establish a tentative reference metric. The feedback is vital, as Clothes4all, with all rules enabled, strives to be as accessible and as universally designed as possible. Originally, we were aiming for more than 20 users. There were, however, a number of obstacles regarding the recruitment of participants and the successful conduction of trials in a remote manner, which explains why we only had the valid data of 11 participants at the end of the project. Even though the participant count is lower than anticipated, it is positive that the user categories cover a wide variety of impairments in the cognitive, motor, and sensory domain. The 
participants used various technical devices, ranging from normal laptops to assistive technologies such as gaze interaction software (e.g. Tobii), specially tailored keyboards, joystick mouse, screen reader software (e.g. Jaws), tactile control switches, and screen magnifier software.

A user trial was composed of two rounds. In the first round, a user had to visit Clothes4all with all rules disabled, i.e. basically meeting a site with various accessibility issues. First, each user had to solve a series of small tasks, as described above. Some tasks ("what is your first impression") required open answers, while others ("how much for returns") checked whether or not a user was able to find or understand a particular piece of information. Then, each user had to fill out a System Usability Scale (SUS), which in turn is a Likert scale consisting of 10 items (questions), each with five answering options, from "disagree vehemently" to "agree completely" [15]. In the second round, the entire process had to be repeated, but this time without accessibility issues, i.e. with all rules enabled.

The users gave Clothes4all with all rules disabled a SUS score average of 59, with a maximum of 78 and a minimum of 38 . This is clearly below the average SUS score, which is 68 [16], but it is an expected result: With all rules disabled, images lack the alt attribute, text like headlines have poor contrast, the top menu bypass is lacking, the specification of the page's language is non-existent, and form elements are coded without labels. This resulted in user comments like "not satisfied with contrast", "problems with Jaws in various browsers", and "not working optimal regarding speech synthesis", as well as a list with issues to be fixed. To our surprise, not a single user filled out the SUS questionnaire during the second round, which can be attributed to the remote (unsupervised) testing procedure. As a consequence, there is unfortunately no SUS score for the accessible version of the Clothes4all.

Before concluding, we underline the following limitations of this study. Due to the small number of participants in the user trials, the validity of the SUS score is limited. Also, the site currently covers only a few accessibility flaws / WCAG criteria and is thus not complete. It can therefore only be called a prototype. Nevertheless, it is fit for its very purpose in education as well as web development and testing, and its flexibility and technical choices lay a proper foundation for future improvements and extension.

\section{Conclusion}

We have built a novel web resource, Clothes4all, which can be used to study various aspects of online accessibility. The application has been developed in a user-centered manner and offers a number of advantages as compared to existing tools. Most importantly, accessibility barriers can be injected into or removed from the site's web pages in a controlled manner, and the site consists of a set of coherent web pages that can be freely used, studied, and extended as desired.

Clothes4all is quite versatile. On the one hand, it can serve as a tool for web developers, testers, and designers to learn about the accessibility of web pages and the connection to assistive technology. On the other hand, it is a valuable means to build better accessibility checkers and validators by in turn testing and validating those tools.

The user trials could confirm that the main ability of Clothes4all to inject accessibility failures into web pages works as expected. The site is thus ready to be used for the development of better tools for testing web accessibility, and it also can be 
employed in educational contexts. Other than that, the results in terms of SUS scores from the user trials were unfortunately limited due to difficulties with participant recruiting and the unsupervised testing process.

\section{Outlook}

Currently, we are working to integrate Clothes4all into the curriculum of selected classes at the University of Oslo and Oslo Metropolitan University, and there is work being done on addressing accessibility and usability issues that were revealed in our user trials.

In the future, we plan to add more accessibility rules, and we hope that the site gets the attention of both students and developers with contributions and code patches as the most desired result. There are multiple ways of extending Clothes4all, by adding one or several languages, by adding more products (clothes) of various categories, by erecting other pages and UI components such as Special Offers, Size and Fabric Details, etc., by modifying existing pages, and similar. The checkout process can be improved upon, and we can add common webshop features, like adding user profiles, saving previous orders, etc. After more rules have been added, a new round with user trials should also be carried out.

There also is a lot of room to develop the site as a learning portal. Among the first features to add is a design system/component library. This is already in development, where reusable components in accessible and inaccessible versions are made available, with explanations for different code, markup, and style choices and possible pitfalls. Adding tutorials, background reading, and training tasks are also among features that could be added in the future, making the site even more useful for training purposes.

\section{References}

[1] Y. Yesilada and S. Harper, Web Accessibility: A Foundation for Research. Springer, 2019.

[2] W3C WAI, "Home," Web Accessibility Initiative (WAI), 2020. https://www.w3.org/WAI/ (accessed Feb. 21, 2020).

[3] W3C WAI, "Web Content Accessibility Guidelines 1.0," May 05, 1999. https://www.w3.org/TR/WAIWEBCONTENT/ (accessed Feb. 21, 2020).

[4] W3C WAI, "Web Content Accessibility Guidelines (WCAG) 2.0,” Dec. 11, 2008. https://www.w3.org/ TR/WCAG20/ (accessed Feb. 21, 2020).

[5] W3C, "Web Content Accessibility Guidelines (WCAG)," 2.1, Jun. 2018. [Online]. Available: https://www.w3.org/TR/WCAG21/.

[6] S. L. Henry, S. Abou-Zahra, and J. Brewer, "The role of accessibility in a universal web," in Proceedings of the 11 th Web for All Conference, Seoul, Korea, Apr. 2014, pp. 1-4, Accessed: Mar. 12, 2021. [Online].

[7] T. Halbach and K. S. Fuglerud, "On Assessing the Costs and Benefits of Universal Design of ICT," in Universal Design 2016: Learning from the Past, Designing for the Future, Aug. 2016, [Online]. Available: http://ebooks.iospress.n1/ISBN/978-1-61499-684-2.

[8] K. S. Fuglerud, "Inclusive design of ICT: The challenge of diversity," University of Oslo, Faculty of Humanities, 2014. doi: 10.13140/2.1.4471.5844.

[9] T. Halbach and I. Tjøstheim, "Towards Reliable Accessibility Assessments of Science Center Exhibits," in Human-Computer Interaction - INTERACT 2019, 2019, pp. 33-41.

[10] United Nations, "Convention on the Rights of Persons with Disabilities." 2006, [Online]. Available: http://www.un.org/disabilities/convention/conventionfull.shtml.

[11] W3C-WAI, "Before and After Demonstration: Overview," 2012. https://www.w3.org/WAI/demos/bad/ (accessed Feb. 21, 2020). 
[12] GDS accessibility team, "Accessibility tools audit results - Overview," 2018. https://alphagov.github.io/ accessibility-tool-audit/ (accessed Apr. 02, 2019).

[13] MediaLT, "Stabile testsider," 2020. https://eksempelsamling.medialt.no/ (accessed Feb. 21, 2020).

[14] ACT Rules Community, "Rules," 2020. https://act-rules.github.io/rules/ (accessed Feb. 24, 2020).

[15] J. Brooke and Others, "SUS-A quick and dirty usability scale," Usability evaluation in industry, vol. 189, no. 194, pp. 4-7, 1996.

[16] J. Sauro, A practical guide to the system usability scale: Background, benchmarks \& best practices. Measuring Usability LLC, 2011. 